EUROPEAN ORGANIZATION FOR NUCLEAR RESEARCH

CERN-EP/98-56 (revised)

April 9, 1998

revised June 22, 1998

\title{
Pseudo-Dirac neutrinos as a potential complete solution to the neutrino oscillation puzzle
}

\author{
A. Geiser1
}

\begin{abstract}
A solution for the neutrino mass and mixing pattern is proposed which is compatible with all available experimental data on neutrino oscillations. This solution involves Majorana neutrinos of the pseudo-Dirac type, i.e. $m_{\text {Majorana }} \ll m_{\text {Dirac }}$. The solar and atmospheric neutrino observations are mainly explained as $\nu_{e}-\nu_{e}^{S}$ and $\nu_{\mu}-\nu_{\mu}^{S}$ oscillations, where S indicates the sterile ("righthanded") partner of each neutrino generation, while the LSND result is interpreted in terms of standard $\nu_{\mu}-\nu_{e}$ oscillations. The resulting constraints on $\nu_{\mu}-\nu_{\tau}$ and $\nu_{\tau}-\nu_{\tau}^{S}$ oscillations are also discussed. This solution leaves room for a hierarchical mass and mixing scheme with a $\nu_{\tau}$ mass in the few $\mathrm{eV}$ range, as favoured by some dark matter scenarios. The apparent conflict with standard Big Bang nucleosynthesis is addressed and the implications for current and future experiments are discussed. It is argued that both short and long baseline accelerator neutrino experiments are needed in order to decide between this solution and other oscillation scenarios.
\end{abstract}

to be published in Phys. Lett. B

PACS codes: 14.60.Pq, 14.60.St

keywords: pseudo-Dirac, neutrino, oscillation, oscillations, sterile, mass

\footnotetext{
${ }^{1}$ CERN, EP Division, CH-1211 Genève 23, Tel. +41-22-7678564, Fax +41-22-7679070, e-mail Achim.Geiser@cern.ch
} 


\section{Introduction}

Currently there is a wealth of unexplained phenomena in neutrino physics which can be interpreted as indications for the existence of neutrino oscillations. The solar neutrino problem [1], lacking a satisfactory astrophysical solution, is commonly attributed to the disappearance of electron (anti)neutrinos into some other neutrino type. Similarly, the atmospheric neutrino anomaly [2] is often interpreted as an indication of neutrino oscillations involving $\nu_{\mu}$ disappearance, with or without partial reappearance as $\nu_{e}$. The LSND experiment [3] claims direct evidence for $\nu_{\mu}-\nu_{e}$ oscillations in a region which is partially unconstrained by other experiments. Furthermore, neutrinos are a prime candidate for a partial solution to the missing dark matter problem [4] if at least one mass eigenstate lies in the eV range. These current indications are summarized in Fig. 1.

Within the framework of 3 generation neutrino mixing, all recent solutions [5] [6] [7] to this neutrino puzzle either discard some of the experimental evidence or have to accept a bad fit to part of the data. Other solutions [8] involve the existence of one additional light sterile neutrino, yielding at least two extra degrees of freedom (mass and mixing angle(s)). Solutions involving more than one such neutrino [9] 10] have often been discarded due to bounds on the number of neutrino types suggested by Big Bang nucleosynthesis [11] [12].

In this paper a solution is presented based on the assumption that neutrinos are of the pseudo-Dirac type, i.e. $m_{\text {Majorana }} \ll m_{\text {Dirac }}$ [13]. This involves the splitting of each neutrino generation into two almost degenerate mass eigenstates, leading to neutrino-antineutrino oscillations with maximal mixing, similar to oscillations in the $K^{0}-\bar{K}^{0}$ system. Since oscillations do not change the handedness, left-handed neutrinos (right handed antineutrinos) will transform into left-handed antineutrinos (right handed neutrinos) which do not participate in weak interactions, and therefore appear sterile.

In contrast to earlier schemes of this kind [10], the neutrino magnetic moments are assumed to be 0, therefore avoiding $\nu_{L} \leftrightarrow \nu_{R}$ transitions. Special emphasis is placed on the case of exactly maximal mixing, which minimizes matter effects. Furthermore, the scheme is extended to include 3 generation flavour mixing, and applied to the most recent experimental data.

With only one parameter (a mass splitting parameter chosen to be universal for simplicity) in addition to the standard 3 generation mixing, all experimental indications for neutrino oscillations can be fully explained in the context of a hierarchical mass and mixing pattern, analogous to the quark sector. In addition, this solution suggests a substantial neutrino contribution to the missing dark matter. A mechanism through which the Big Bang nucleosynthesis bounds mentioned earlier can be evaded is discussed in the appendix.

\section{The pseudo-Dirac neutrino formalism}

The most general neutrino mass term (Dirac-Majorana mass term) that can be added to the standard model Lagrangian leads, for one neutrino generation, to the (Majorana) mass eigenstates [13] [14]

$$
m_{I, I I}=\left|\frac{1}{2}\left[\left(m_{L}+m_{R}\right) \pm \sqrt{\left(m_{L}-m_{R}\right)^{2}+4 m_{D}^{2}}\right]\right|
$$


where $m_{D}$ is the Dirac mass, and $m_{L}, m_{R}$ are the left and right handed Majorana masses, and to a mixing angle

$$
\tan 2 \theta=2 m_{D} /\left(m_{R}-m_{L}\right)
$$

between these two states.

The standard Dirac case is obtained by setting $m_{L}=m_{R}=0$, while $m_{D}=0$ leads to pure Majorana neutrinos. The limit $m_{R} \gg m_{D}, m_{L}=0\left(\theta=m_{D} / m_{R} \ll 1\right)$ yields the mass eigenstates $m_{I}=m_{D}^{2} / m_{R}, m_{I I} \simeq m_{R}$ which corresponds to the well-known see-saw model [15], where $m_{D}$ is assumed to be of the order of the quark and charged lepton masses, and $m_{R}$ is a large mass of the order of some unification scale. This forces the mass of the "left-handed" neutrino $\left(m_{I}\right)$ to be small, and makes the "right-handed" neutrino $\left(m_{I I}\right)$ very heavy, therefore decoupling it from interactions in the accessible energy range.

Finally, the limit $m_{D} \gg m_{L}, m_{R}\left(\theta \simeq 45^{\circ}\right)$ yields almost degenerate mass eigenstates $m_{I, I I} \simeq m_{D}$, with close to maximal mixing arising in a "natural" way. This case, which is known as the pseudo-Dirac case, will be pursued further here. Taking $m_{L}=m_{R}=\delta m$ for simplicity one obtains

$$
m_{I, I I}=m_{D} \pm \delta m, \quad \theta=45^{\circ} .
$$

In this case, the mass eigenstates can be written in terms of the neutrino/antineutrino eigenstates of the weak interactions as

$$
\left|\nu_{I}>=\frac{1}{\sqrt{2}}(|\nu>+| \bar{\nu}>), \quad\right| \nu_{I I}>=\frac{1}{\sqrt{2}}(|\nu>-| \bar{\nu}>),
$$

a pattern which is similar to the one in the $K^{0}-\bar{K}^{0}$ meson system. As in the $K^{0}$ case, this yields maximal oscillations between particle and antiparticle states if pure neutrinos or antineutrinos are produced. Since, up to a small mass effect, neutrinos are emitted lefthanded and oscillations cannot change the handedness (angular momentum conservation) they will oscillate into left-handed antineutrinos which appear sterile. The same is true for right-handed antineutrinos oscillating into right-handed neutrinos. Thus phenomenologically one obtains active-sterile neutrino oscillations with

$$
\Delta m^{2}=\left(m_{D}+\delta m\right)^{2}-\left(m_{D}-\delta m\right)^{2}=4 m_{D} \delta m
$$

and maximal mixing $\left(\sin ^{2} 2 \theta=1\right)$.

This can easily be generalized to 3 generations. Neglecting CP violation, the resulting mixing matrix is a real $6 \times 6$ matrix in principle. Here, we simplify it with the hypothesis that the oscillations between different generations and the active-sterile oscillations decouple, thus yielding the standard 3 generation mixing matrix $U_{\alpha i}(\alpha=e, \mu, \tau, i=1,2,3)$, equivalent to the CKM matrix [16], plus maximal active-sterile mixing for each generation

$$
\left|\nu_{\alpha}>=U_{\alpha i} \frac{1}{\sqrt{2}}\left(\left|\nu_{i, I}>+\right| \nu_{i, I I}>\right), \quad\right| \bar{\nu}_{\alpha}>=U_{\alpha i} \frac{1}{\sqrt{2}}\left(\left|\nu_{i, I}>-\right| \nu_{i, I I}>\right) .
$$

The above discussion remains valid to good approximation for $m_{L} \neq m_{R}$ as long as both terms are very small, and in particular for $m_{L}=0$. Since $m_{R}$ mass terms can be introduced ad hoc as bare mass terms without destroying gauge invariance, no attempt is made at this stage to clarify the origin of the Majorana masses. Phenomenologically, two cases are 
considered: (1) same $\delta m_{i}=\varepsilon \times M$ for each generation, where $M=1 \mathrm{eV}$ is an arbitrary fixed reference mass, and (2) mass dependent splitting $\delta m_{i}=\varepsilon \times m_{D i}$. The mass pattern generated by these scenarios is therefore given by $m_{D i} \simeq m_{1}, m_{2}, m_{3}$ for the 3 generations, and by the single additional parameter $\varepsilon \ll 1$ fixing the mass fine splittings.

\section{The neutrino oscillation solution}

To find a simple general solution to the neutrino oscillation puzzle described in the introduction the following further assumptions are made, in analogy to the quark sector:

- The mass structure is hierarchical, i.e. $m_{1} \ll m_{2} \ll m_{3}$.

- Mixing between adjacent generations is small $\left(\theta_{12}\right.$ and $\left.\theta_{23}\right)$ and mixing between far generations is negligible $\left(\theta_{13} \simeq 0\right)$. Flavour eigenstates can therefore be approximately identified with mass eigenstates $(1 \simeq e, 2 \simeq \mu, 3 \simeq \tau)$.

Including $\varepsilon$ this yields a total of six free parameters $\left(m_{i}(i=1,2,3), \varepsilon, \theta_{12}, \theta_{23}\right)$.

The $\Delta m^{2}$ values relevant for oscillations are $\Delta m_{12}^{2}, \Delta m_{23}^{2}$ for conventional mixing, and $\Delta m_{I / I I, i}^{2}=4 m_{i} M \varepsilon$ for case (1) $\left(\Delta m_{I / I I, i}^{2}=4 m_{i}^{2} \varepsilon\right.$ for case (2)) for active-sterile oscillations. In the simplified case of only two contributing flavour and mass eigenstates, the probability of a neutrino of type $\alpha$ to oscillate into type $\beta$ is given by the well known formula

$$
P_{\alpha \beta}=\sin ^{2} 2 \theta_{\alpha \beta} \sin ^{2}\left(1.27 \Delta m_{\alpha \beta}^{2} L / E\right)
$$

where $\mathrm{L}$ is the distance travelled in $\mathrm{km}, \mathrm{E}$ is the neutrino energy in $\mathrm{GeV}$, and $\Delta m_{\alpha \beta}^{2}$ is expressed in $\mathrm{eV}^{2}$.

In the following, a complete solution for the six free parameters is derived based on all known experimental evidence (table 1). A graphical representation of this solution is shown in Fig. 11.

\subsection{LSND}

The LSND result [3] is interpreted as direct $\nu_{\mu}-\nu_{e}$ oscillations. The large $\Delta m^{2}$ region $\left(\Delta m^{2}>10 \mathrm{eV}^{2}\right)$ is excluded by NOMAD [17], CCFR [18], BNL 734 [19], and BNL E766 [20]. The latter constrains also the island around $\Delta m^{2} \sim 6 \mathrm{eV}^{2}$ advocated by Caldwell [21]. The remaining region $\left(\Delta m^{2} \leq 2 \mathrm{eV}^{2}\right)$ is fully consistent with, and even slightly favoured by, the recent KARMEN1 limits [22] partially based on a small $(\sim 1 \sigma)$ positive result. At large $\sin ^{2} 2 \theta$ the result is constrained by reactor experiments, including Gösgen [23], Krasnoyarsk [24], Chooz [25] and Bugey [26]. Note that the Bugey result, which yields the most stringent limit, is also based on a small (up to $1.5 \sigma$ ) positive effect [26], fully consistent with the limits from the other reactor experiments. Inspired by this effect we choose a reference solution $\Delta m_{12}^{2} \sim 0.5 \mathrm{eV}^{2}, \sin ^{2} 2 \theta_{12} \sim 2 \times 10^{-2}$. However, no significant lower constraint on $\sin ^{2} 2 \theta$ can be derived from the Bugey result, allowing $\sin ^{2} 2 \theta_{12}$ to be as small as $2 \times 10^{-3}$ for $\Delta m_{12}^{2} \sim 2 \mathrm{eV}^{2}$. 


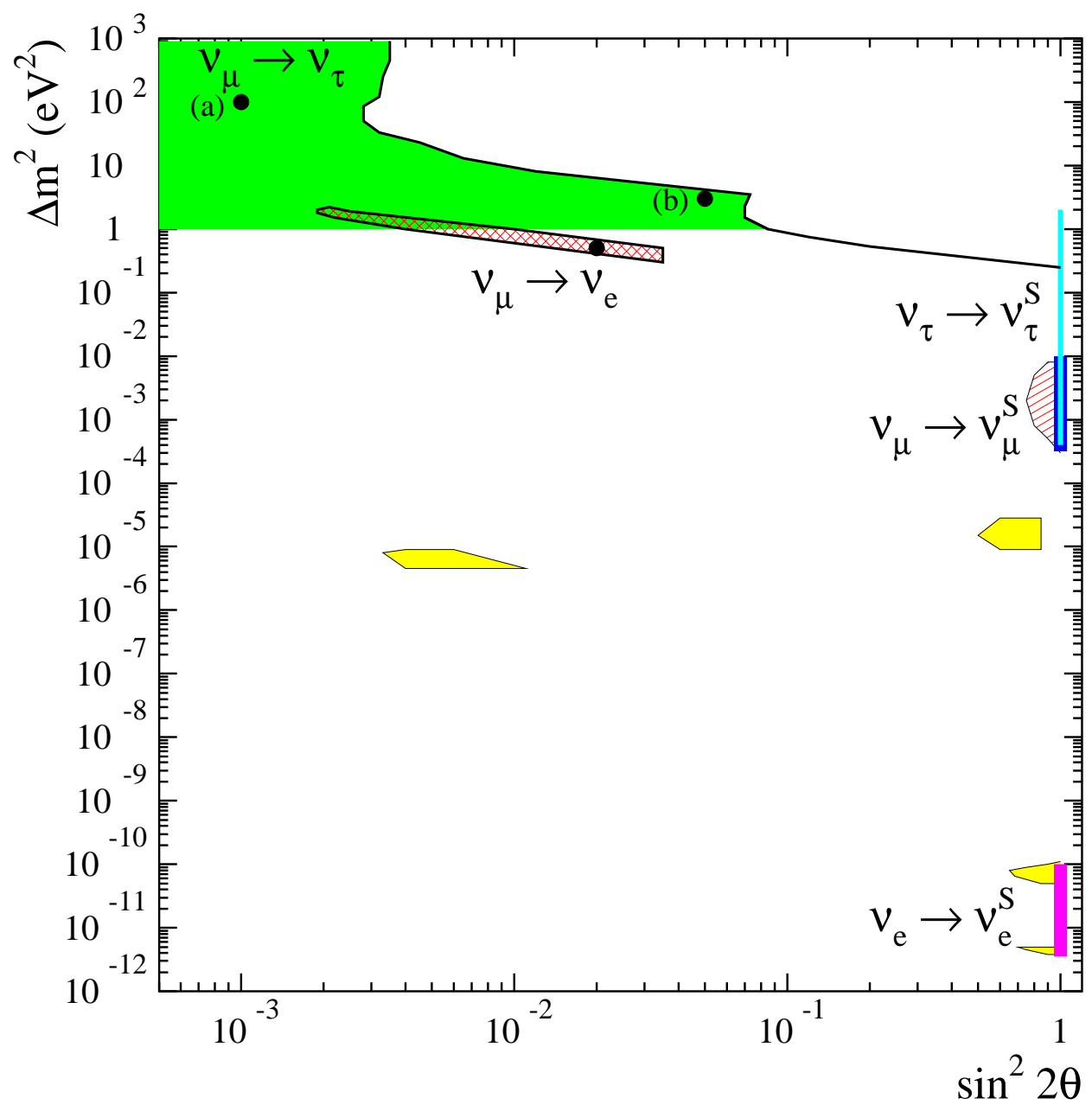

Figure 1: Current indications for neutrino oscillations in the $\sin ^{2} 2 \theta$ vs. $\Delta m^{2}$ plane, assuming dominance of two flavour oscillations in each case. The figure shows the $90 \%$ c.l. $\nu_{\mu} \rightarrow \nu_{e}$ region favoured by LSND (double hatched), including constraints from BNL 776 and Bugey, the Superkamiokande $\nu_{\mu}$ disappearance solution to the atmospheric neutrino problem (hatched), the 3 mutually exclusive $95 \%$ c.l. solutions to the solar neutrino problem (light shaded), and the $90 \%$ c.l. limit on $\nu_{\mu} \rightarrow \nu_{\tau}$ (black line). Also shown is the parameter space of the proposed solutions for $\nu_{X} \rightarrow \nu_{X}^{S}$ (bars at $\sin ^{2} 2 \theta=1$ ), $\nu_{\mu} \rightarrow \nu_{\tau}$ (dark shaded) and $\nu_{\mu} \rightarrow \nu_{e}$ (double hatched, identical to LSND region). The reference solutions described in the text are also indicated (black circles). 


\subsection{Solar neutrinos}

The solar neutrino problem is interpreted as $\nu_{e}-\nu_{e}^{S}$ oscillations with $\sin ^{2} 2 \theta_{e S}=1$. This yields an average expected suppression factor of 0.5 , slightly reduced further by a $\mathrm{O}(1 \%)$ correction from $\nu_{e}-\nu_{\mu}$ oscillations. Energy dependent oscillations have to be introduced in order to describe the observed suppression factors [27] with respect to the standard solar model [28] of $0.51 \pm 0.05$ for the Gallium experiments [29], $0.27 \pm 0.02$ for the Homestake experiment [30], and $0.39 \pm 0.03$ for the water Cerenkov detectors 31] 32. Since the MSW solution [27] 33] is irrelevant for maximal mixing, the vacuum solution [27] 34] is the only possible one. This yields $\Delta m_{I / I I, 1}^{2} \sim 5 \times 10^{-12}-10^{-10} \mathrm{eV}^{2}$.

\subsection{Atmospheric neutrinos}

The atmospheric neutrino anomaly is interpreted as $\nu_{\mu}-\nu_{\mu}^{S}$ oscillations with $\sin ^{2} 2 \theta_{\mu S}=1$. A small effect from $\nu_{\mu}-\nu_{e}$ and $\nu_{\mu}-\nu_{\tau}$ oscillations should be added. The $\Delta m^{2}$ for $\nu_{e}$ disappearance $\left(\Delta m_{I / I I, 1}^{2}\right)$ is too small to have any influence, so electron-like events are expected to be slightly enhanced by $\nu_{\mu}-\nu_{e}$ oscillations without L/E dependence. On the other hand, muon-like events are depleted mainly by $\nu_{\mu}-\nu_{\mu}^{S}$ oscillations, with a small L/E independent suppression from $\nu_{\mu}-\nu_{e}$, and another small but potentially complicated contribution from $\nu_{\mu}-\nu_{\tau}$ followed by $\nu_{\tau}-\nu_{\tau}^{S}$. Using the LSND reference solution quoted above, an L/E independent suppression would imply a value of $R=\frac{\mu / e_{\text {measured }}}{\mu / e_{\text {expected }}}$ of 0.49 or lower (if $\nu_{\mu}-\nu_{\tau}$ is significant). The observed average suppression of only about 0.63 [32] is therefore an indication for non-maximal $\nu_{\mu}$ suppression due to dependence on $\mathrm{L} / \mathrm{E}$. Indeed such a dependence is observed indirectly through a dependence on the zenith angle by Kamiokande [35] and Superkamiokande [36]. Reinterpretation of the Superkamiokande result in terms of maximal

$\nu_{\mu}-\nu_{\mu}^{S}$ oscillations suggests a value of $\Delta m_{I / I I, 2}^{2}$ in the range $3 \times 10^{-4}-10^{-2} \mathrm{eV}^{2}$ [37]. The detailed consideration of matter effects can potentially induce a slight upward shift of this range [38]. Such low $\Delta m^{2}$ values can also explain why the suppression is stronger for the water Cerenkov detectors [35] 36] than for some of the iron detectors 39] which have a higher energy threshold. Experiments looking for upward going muons yield inconclusive results so far 40] 41, but are not inconsistent with the $\nu_{\mu}-\nu_{\mu}^{S}$ interpretation.

\subsection{Dark matter}

The potential contribution of neutrinos to hot dark matter is given by $\Omega_{\nu}=\Sigma m_{\nu} /\left(92 h^{2} \mathrm{eV}\right)$ [42, where $\Omega_{\nu}$ is the neutrino energy density parameter expressed as the fraction of the critical density $\Omega=1$ needed to close the universe, $h$ is the dimensionless renormalized Hubble constant $\left(0.5<h<0.85\right.$ [43]) and $\Sigma m_{\nu}$ is the sum over the masses of all neutrino types. For pseudo-Dirac neutrinos each neutrino flavour enters this sum twice if its sterile counterpart reaches thermal equilibrium in the early universe, and at least once otherwise. In the hierarchical mass pattern advocated here this contribution arises mainly from $\tau$ neutrinos [44. No direct evidence for $\nu_{\mu}-\nu_{\tau}$ oscillations exists so far in the context of the pseudo-Dirac solution. Constraints are imposed by the exclusion regions obtained by accelerator experiments [17 45 [46] 47. Two typical solutions close to the range accessible by current experiments and relevant to the dark matter problem could be (a) $\Delta m_{23}^{2}=100 \mathrm{eV}^{2}\left(m_{3}=10 \mathrm{eV}\right)$ 
with $\sin ^{2} 2 \theta_{23}=10^{-3}$, and (b) $\Delta m_{23}^{2}=3 \mathrm{eV}^{2}\left(m_{3} \simeq 2 \mathrm{eV}\right)$ with $\sin ^{2} 2 \theta_{23}=5 \times 10^{-2}$. Solution (a), with its very small mixing, would have virtually no effect on any of the current measurements with positive indications for oscillations, but yields a large hot dark matter component $\left(\Omega_{\nu} \sim 0.4\right.$ for $\left.h \sim 0.7\right)$ which is disfavoured by most current dark matter scenarios 4 . The associated $\Delta m_{23}^{2}$ could however be lowered to any value larger than $1 \mathrm{eV}^{2}$ (mass hierarchy limit), corresponding to $\Omega_{\nu}>0.03$, without significantly changing the conclusions. Alternatively, it could be raised up to the extreme limit from pure hot dark matter models of about $(30 \mathrm{eV})^{2}$ [44]. Solution (b) would yield a small but significant contribution of about $5 \%$ to the atmospheric neutrino suppression, with only a moderate hot dark matter contribution $\left(\Omega_{\nu} \sim 0.1\right.$ for $\left.h \sim 0.7\right)$.

\subsection{The mass fine splitting}

The $\Delta m^{2}$ for $\nu_{\tau}-\nu_{\tau}^{S}$ oscillations is essentially unconstrained by current data. Since $m_{2} \sim 1 \mathrm{eV}$ (LSND), the atmospheric neutrino solution yields $\varepsilon \sim 10^{-4}-2 \times 10^{-3}$ for both case (1) and case (2). Folding this with the $\Delta m_{23}^{2}$ parameter space spanned by $\nu_{\mu}-\nu_{\tau}$ solutions (a) and (b), $\Delta m_{I / I I, 3}^{2}$ could be anywhere in the range $\sim 10^{-3}-1 \mathrm{eV}^{2}$. In combination with $\nu_{\mu}-\nu_{\tau}$ oscillations this could yield a small contribution to the angular effect observed in the atmospheric neutrino anomaly.

Finally, the above $\varepsilon$ value applied to the solar neutrino result predicts $m_{1}$ in the range $10^{-9}-10^{-6} \mathrm{eV}$ for case (1), and $10^{-5}-10^{-3} \mathrm{eV}$ for case (2). This mass and mixing pattern is in agreement with the nonobservation of neutrinoless double $\beta$ decay 48 .

Table 1: Parameters of the pseudo-Dirac neutrino solution for $\varepsilon=10^{-4}-2 \times 10^{-3}$ (cases (1) and (2) of section 3.5 combined). $m$ refers to the Dirac mass of each neutrino generation and $\Delta m^{2}$ to either the mass fine splitting inside each generation, or the mass difference squared between generations where appropriate. $\sin ^{2} 2 \theta$ refers to the associated mixing angle. Only the entries in the $m$ and $\sin ^{2} 2 \theta$ columns are independent parameters, $\Delta m^{2}$ being fixed by $m$ and $\varepsilon$.

\begin{tabular}{|l|c|c|c|}
\hline generation & $m(\mathrm{eV})$ & $\Delta m^{2}\left(\mathrm{eV}^{2}\right)$ & $\sin ^{2} 2 \theta$ \\
\hline $1(" e ")$ & $10^{-9}-10^{-3}$ & $5 \times 10^{-12}-10^{-10}$ & 1 \\
\hline $2(" \mu ")$ & $0.5-1.7$ & $3 \times 10^{-4}-10^{-2}$ & 1 \\
\hline $3(" \tau ")$ & $1-30$ & $4 \times 10^{-4}-2$ & 1 \\
\hline $1 \leftrightarrow 2$ & - & $0.3-3$ & $0.002-0.035$ \\
\hline $2 \leftrightarrow 3$ & - & $1-30$ & $<0.07$ \\
& & or $30-900$ & $<0.0035$ \\
\hline $1 \leftrightarrow 3$ & - & $1-900$ & negligible \\
\hline
\end{tabular}

\section{Alternative solutions}

Only two of the critical experimental inputs are so far not cross-checked independently by at least one other experiment: The LSND result [3], and the Homestake result [30] 
for solar neutrinos. The former fixes the $\nu_{\mu}-\nu_{e}$ oscillation parameters and, if disproven, would essentially remove any lower constraint on $\Delta m_{12}^{2}$. Comparison of the latter to the other solar neutrino experiments yields the main evidence for the energy-dependence of the solar neutrino deficit. A 30\% increase of the Homestake neutrino flux measurement (not compatible with the quoted error), and a reduction of the predicted $B^{8}$ solar neutrino flux by about $20 \%$ (which is inside the currently discussed error margin 49]) would make all solar neutrino measurements compatible with a flat suppression factor of about 0.5 (see also [5] [7]). This would remove the constraint on $\Delta m_{I / I I, 1}^{2}$ which, ignoring Big Bang nucleosynthesis implications, could then be as large as $10^{-4} \mathrm{eV}^{2}$ (bounded by its potential effect on the atmospheric neutrino anomaly). In order to fix the parameters of the pseudo-Dirac model the verification of LSND by KARMEN2 [22] and later by other experiments [50] is therefore crucial, and a verification of the energy dependence of the solar neutrino suppression, e.g. by Borexino [51], is highly desirable. Note that the azimuthal dependence of the atmospheric neutrino suppression, which is observed by only one type of experiment, is not an a priori independent experimental input in this context, but rather a prediction of the model based on the observed non-maximal $\nu_{\mu}$ suppression.

\section{$5 \quad$ Predictions for current and future experiments}

The solution of the neutrino oscillation puzzle proposed in this paper can be checked by several current and future experiments.

Since the solar neutrino problem is solved via $\nu_{e}$ disappearance into sterile neutrinos, SNO 52 should not observe an enhancement of the $\mathrm{NC} / \mathrm{CC}$ ratio as it would in the case of $\nu_{e} \rightarrow \nu_{\mu}$. No day/night effect should be present, but a small seasonal effect might be expected (vacuum solution).

The atmospheric neutrino anomaly should be confirmed, including the azimuthal dependence of the $\mu / e$ suppression. Since it is mainly due to $\nu_{\mu}$ disappearance into sterile neutrinos, the muon deficit (rather than electron excess) should be confirmed, and the $\nu_{\mu} \mathrm{NC} / \mathrm{CC}$ ratio should not be enhanced. Experiments looking at upward going muons [41] should also see an effect, and current reactor experiments testing $\nu_{\mu}-\nu_{e}$ oscillations 250 53 should continue to observe negative or marginal results. Long baseline accelerator experiments [54 55 [56] should not observe a strong $\nu_{\tau}$ appearance effect, but should observe maximal $\nu_{\mu}$ disappearance if the $\nu_{\mu}-\nu_{\mu}^{S}$ oscillations lie in the experimentally accessible range. Unfortunately, the low $\Delta m^{2}$ range $\left(\Delta m^{2}<10^{-3} \mathrm{eV}^{2}\right)$ allowed by Superkamiokande [32] can not be fully tested by any of the currently planned long baseline experiments.

However, ICARUS [54] and MINOS [55], if sensitive enough, should observe energy independent $\nu_{\mu}-\nu_{e}$ oscillations, whose (small) rate should finally settle the value of $\sin ^{2} 2 \theta$ for the LSND result.

$\nu_{\mu}-\nu_{\tau}$ oscillations should be observed in short baseline accelerator experiments within the next decade. If not by the current experiments NOMAD and CHORUS [45], then by future more sensitive experiments [57] with a slightly lower $\Delta m^{2}$ threshold. $\nu_{\mu}-\nu_{\tau}$ solution (b) would also be within the reach of the long baseline $\tau$ appearance experiments [54 [56], and could possibly be confused with a signal at low $\Delta m^{2}$ in the atmospheric neutrino region. Therefore both short and long baseline experiments are needed to unambigously confirm or 
rule out this oscillation scenario. Since $m_{3}$ is of order eV or more (hierarchy), $\tau$ neutrinos should make up a significant part of the missing dark matter.

If $\nu_{\mu}-\nu_{\tau}$ oscillations would be discovered with sizeable mixing, $\nu_{\tau}-\nu_{\tau}^{S}$ disappearance could be verified in the long term by comparing the $\tau$ production rates in short, medium, and long baseline experiments. Some corrections to the atmospheric neutrino spectrum might also be observable.

Lastly, if this pseudo-Dirac neutrino solution would be confirmed and some of its parameters measured more precisely, the complicated interplay between lepton number violating active-sterile neutrino oscillations, matter effects, and Big Bang nucleosynthesis (see appendix) could be used to further constrain the remaining parameters.

\section{Appendix: Compatibility with Big Bang nucleosynthe- sis}

As is well known [58], the ratio of deuterium to hydrogen $(\mathrm{D} / \mathrm{H})$ and the fraction of $\mathrm{He}^{4}$ in the universe $(\mathrm{Y})$ is determined in the Big Bang model by the ratio of neutrons to protons at the time of the "weak freeze out", i.e. the time when the reactions $n+e^{+} \leftrightarrow p+\bar{\nu}_{e}$ and $p+e^{-} \leftrightarrow n+\nu_{e}$ proceed too slowly compared to the expansion rate of the universe to keep $\mathrm{n} / \mathrm{p}$ at its thermal equilibrium value. The effective number of light neutrino flavours $N_{\nu}$ enters as a contribution to the energy density, which in turn influences the expansion rate. From this effect, a typical upper limit derived from current Big Bang nucleosynthesis (BBN) models is $N_{\nu}<3.1$ @ 95\% c.l. [58] with a central value of about 2.3, only marginally compatible with the standard model $N_{\nu}=3$.

Active-sterile neutrino mixing can influence this rate in two ways: If active-sterile oscillations occur during thermal equilibrium, i.e. significantly before weak freeze out, the sterile neutrinos will make an additional contribution to the energy density at freeze out, and therefore increase the expansion rate, effectively behaving like additional light neutrino generations. On the other hand, depletion of $\nu_{e}$ due to $\nu_{e} \rightarrow \nu_{e}^{S}$ during freeze-out reduces the reaction rate for $\mathrm{n} / \mathrm{p}$ interchange. In both cases, the predicted $\mathrm{D} / \mathrm{H}$ and $\mathrm{Y}$ values will be larger than in the standard $N_{\nu}=3$ case, and therefore even less compatible with the observed values which yield the limit quoted above.

The $\Delta m^{2}$ of $10^{-10} \mathrm{eV}^{2}$ or lower for vacuum $\nu_{e}-\nu_{e}^{S}$ oscillations yields an oscillation time scale which is significantly longer than the freeze out time scale. The oscillation effect is thus negligible for practical purposes [1]. Both $\nu_{\mu}-\nu_{\mu}^{S}$ and $\nu_{\tau}-\nu_{\tau}^{S}$ oscillations approximately fall in the mass range $10^{-3}-10^{-1} \mathrm{eV}^{2}$ for which the BBN implications are discussed in ref. [12]. Ignoring non standard model effects such as lepton number violation, each of these contribute 1 unit to $N_{\nu}$ if $\Delta m^{2}$ is at the upper edge of this range, and slightly less than one unit if $\Delta m^{2}$ is of order $10^{-3}-10^{-4}$ [1], yielding $N_{\nu} \sim 4.6-5$.

However, matter effects induced by a significant initial lepton number asymmetry $\left(10^{-3}\right.$ or larger) can almost fully suppress the $\nu_{\mu}-\nu_{\mu}^{S}$ and $\nu_{\tau}-\nu_{\tau}^{S}$ oscillations during thermal equilibrium [59], therefore avoiding any contribution to the effective number of neutrinos.

Moreover, such an asymmetry can be created by the (lepton number violating) activesterile oscillations themselves [60], if small inter-generation oscillations (i.e. $\nu_{\tau}-\nu_{\mu}^{S}$ ) are 
added. The quantitative treatment of the $\nu_{\mu}-\nu_{\mu}^{S}$ case in ref. 61 is qualitatively also applicable to $\nu_{\tau}-\nu_{\tau}^{S}$ oscillations. Interestingly, the parameters needed to create a lepton number asymmetry which suppresses $\nu_{\mu}-\nu_{\mu}^{S}$ and $\nu_{\tau}-\nu_{\tau}^{S}$ oscillations until they become irrelevant for BBN largely overlap with the parameter space suggested by the pseudo-Dirac solution (table —). For example, taking $\Delta m_{I / I I, 2}^{2}=10^{-3} \mathrm{eV}^{2}, \Delta m_{12}^{2}=1 \mathrm{eV}^{2}\left(\varepsilon=3 \times 10^{-3}\right.$ for case (1)) and adding $\nu_{\tau}-\nu_{\mu}^{S}$ or $\nu_{\tau}-\nu_{e}^{S}$ oscillations with $\sin ^{2} 2 \theta \sim 10^{-6}$, which have a negligible effect on the phenomenology of the pseudo-Dirac model, almost full $\nu_{X}-\nu_{X}^{S}$ suppression $(X=\mu, \tau)$ is obtained for $\Delta m_{23}^{2}>3 \mathrm{eV}^{2}$, which is just what is required from the hierarchy assumption.

Finally, with a similar set of parameters, the "reprocessing" of the initial lepton number asymmetry into an effective excess of $\nu_{e}$ over $\overline{\nu_{e}}$ and a corresponding asymmetry in the $n \rightarrow p$ and $p \rightarrow n$ reaction rates during BBN can induce a reduction of the effective number of neutrinos by as much as 0.5 [62]. This yields the lower bound $N_{\nu} \geq 2.5$, compatible with the current limit. Furthermore, the systematic error on the experimental input for $\mathrm{Y}$ and $\mathrm{D} / \mathrm{H}$ is a matter of ongoing discussion [63], which could contribute to loosening the BBN constraint.

In conclusion, the apparent conflict between the BBN limit and 3 additional sterile neutrinos can be evaded through the lepton number violating (i.e. non standard model) effects caused by active-sterile neutrino oscillations.

\section{Acknowledgements}

Constructive discussions with L. Camilleri, Ch. Cardall, J. Ellis, R. Foot, E. Nagy, U. Stiegler and R. Volkas are gratefully acknowledged.

\section{References}

[1] see e.g. V. Castellani et al., Phys. Rep. 281 (1997) 309.

[2] see e.g. T.K. Gaisser, in Neutrino '96, Proceedings of the 17th International Conference on Neutrino Physics and Astrophysics, Helsinki, Finland, June 13, 1996, ed. by K. Enqvist, K. Huitu and J. Maalampi, World Scientific (1997), p. 211.

[3] LSND Collaboration, C. Athanassopoulos et al., Phys. Rev. C54, 2685 (1996);

LSND Collaboration, C. Athanassopoulos et al., Phys. Rev. Lett. 77, 3082 (1996);

LSND Collaboration, C. Athanassopoulos et al., LA-UR-97-1998, UCRHEP-E191, submitted to Phys. Rev. C; UCRHEP-E197, submitted to Phys. Rev. Lett.

[4] see e.g. J. Primack and A. Klypin, Nucl. Phys. Proc. Suppl. 51 B (1996) 30.

[5] A. Acker and S. Pakvasa, Phys. Lett. B 397 (1997) 209;

P.F. Harrison, D.H. Perkins, and W.G. Scott, Phys. Lett. B 349 (1995) 137; Phys. Lett. B 349 (1997) 186;

E. Torrente-Lujan, Phys. Lett. B 389 (1996) 557. 
[6] C.Y. Cardall and G.M. Fuller, Phys. Rev. D 53 (1996) 4421;

K.S. Babu, J.C. Pati, and F. Wilczek, Phys. Lett. B 359 (1995) 351;

G.L. Fogli, E. Lisi, and D. Montanino, Phys. Rev. D 49 (1994) 3626; Phys. Rev. D 54 (1996) 2048; G.L. Fogli, E. Lisi, and G. Scioscia, Phys. Rev. D 52 (1995) 5334; Phys. Rev. D 56 (1997) 3081; G.L. Fogli et al., Phys. Rev. D 56 (1997) 4365;

H. Minakata, Phys. Lett. B 356 (1995) 61;

S.M. Bilenky et al., Phys. Lett. B 356 (1995) 273; Phys. Rev. D 54 (1996) 1881.

[7] G. Conforto et al., Astropart. Phys. 5 (1996) 147;

S. Barshay and P. Heiliger, Astropart. Phys. 6 (1997) 323.

[8] D.O. Caldwell and R.N. Mohapatra, Phys. Rev. D 48 (1993) 3259; Phys. Rev. D 50 (1994) 3477;

J.T. Peltoniemi and J.W.F. Valle, Nucl. Phys. B 406 (1993) 409;

J.T. Peltoniemi, D. Tommasini and J.W.F. Valle, Phys. Lett. B 298 (1993) 383;

H. Minakata, Phys. Rev. D 52 (1995) 6630;

D. Suematsu, Phys. Lett. B 392 (1997) 413;

Z.G. Berezhiani and R.B. Mohapatra, Phys. Rev. D 52 (1995) 6607;

J.J. Gomez-Cadenas and G.M. Gonzalez-Garcia, Z. Phys. C 71 (1996) 1996.

[9] R. Foot and R.R. Volkas, Phys. Rev. D 52 (1995) 6595;

R. Foot, Mod. Phys. Lett. A 9 (1994) 169;

J.P. Bowes and R.R. Volkas, hep-ph/9804310, to appear in J. Phys. G.

[10] M. Kobayashi, C.S. Lim and M.M. Nojiri, Phys. Rev. Lett. 67 (1991) 1685;

C. Giunti, C.W. Kim and U.W. Lee, Phys. Rev. D 46 (1992) 3034.

[11] X. Shi, D.N. Schramm and B.D. Fields, Phys. Rev. D 48 (1993) 2563;

K. Enqvist, K. Kainulainen and M. Thomson, Nucl. Phys. B 373 (1992) 498.

[12] C.Y. Cardall and G.M. Fuller, Phys. Rev. D 54 (1996) 1260.

[13] L. Wolfenstein, Nucl. Phys. B 186 (1981) 147;

S.M. Bilenky and B.M. Pontecorvo, Yad. Fiz. 38 (1983) 415 (Sov. J. Nucl. Phys. 38 (1983) 248).

[14] G. Gelmini and E. Roulet, Rep. Prog. Phys. 58 (1995) 1207.

[15] J. Ellis and M. Karliner, Phys. Lett. B 213 (1988) 73;

D.B. Kaplan and A. Manohar, Nucl. Phys. B 310 (1988) 527.

[16] N. Cabibbo, Phys. Rev. Lett 10 (1963) 531; M. Kobayashi and T. Maskawa, Progr. Th. Phys. 49 (1973) 652;

F.J. Gilman, K. Kleinknecht and B. Renk, in Review of Particle Physics, Phys. Rev. D 54 (1996), p. 94.

[17] V. Valuev (NOMAD Collaboration), proceedings of the International Europhysics Conference on High Energy Physics, Jerusalem, Israel, 19-26 August 1997, to be published. 
[18] CCFR Collaboration, A. Romosan et al., Phys. Rev. Lett. 78 (1997) 2912.

[19] L.A. Ahrens et al., Phys. Rev. D 31 (1985) 2732.

[20] L. Borodowsky et al.,Phys. Rev. Lett. 68 (1992) 274.

[21] D.O. Caldwell, in Neutrino '96 [2], p. 182.

[22] J. Kleinfeller (KARMEN Collaboration), Nucl. Phys. Proc. Suppl. B 48 (1996) 207;

KARMEN Collaboration, B. Armbruster et al., Phys. Rev. C 57 (1998) 3414, and references therein;

K. Eitel (KARMEN Collaboration), CERN Particle Physics seminar, Febr. 17, 1998.

[23] G. Zacek et al., Phys. Rev. D 34 (1986) 2621.

[24] G.S. Vidyakin et al., JETP Lett. 59 (1994) 237.

[25] CHOOZ Collaboration, M. Apollonio et al., Phys. Lett. B 420 (1998) 397.

[26] B. Achkar et al., Nucl. Phys. B 434 (1995) 503.

[27] N. Hata and P. Langacker, Phys. Rev. D 56 (1997) 6107.

[28] J.N. Bahcall and M.H. Pinsonneault, Rev. Mod. Phys. 67 (1995) 781.

[29] GALlEX Collaboration, P. Anselmann et al., Phys. Lett. B 327 (1994) 377; Phys. Lett. B 342 (1995) 440; W. Hampel et al., Phys. Lett. B 388 (1996) 384;

SAGE Collaboration, J.N. Abdurashitov et al., Phys. Lett. B 328 (1994) 234; Nucl. Phys. B 38 (1995) 60; Phys. Rev. Lett. 77 (1996) 4708.

[30] B.T. Cleveland et al., Nucl. Phys. B (Proc. Suppl.) 38 (1995) 47, and references therein.

[31] Kamiokande Collaboration, Y. Fukuda et al., Phys. Rev. Lett. 77 (1996) 1683.

[32] Y. Totsuka (Superkamiokande results), Proceedings of the Int. Lepton-Photon Symposium, Hamburg 1997 (in press).

[33] L. Wolfenstein, Phys. Rev. D 17 (1978) 2369; Phys. Rev. D 20 (1979) 2634;

S.P. Mikheyev and A.Y. Smirnov, Sov. J. Nucl. Phys. 42 (1985) 913; Nuovo Cimento 9C (1986) 17.

[34] P.I. Krastev and S.T. Petcov, Phys. Rev. Lett. 72 (1994); Phys. Rev. D 53 (1996) 1665.

[35] Kamiokande Collaboration, Y. Fukuda et al., Phys. Lett. B 335 (1994) 237;

IMB Collaboration, R. Becker-Szendy et al., Phys. Rev. D 46 (1992) 237.

[36] Super-Kamiokande Collaboration, Y. Fukuda et al., hep-ex/9803006 and hepex/9805006.

[37] R. Foot, R.R. Volkas and O. Yasuda, Phys. Rev. D (1998) R1345. 
[38] R. Foot, R.R. Volkas and O. Yasuda, TMUP-HEL-9801,UM-P-98/04,RCHEP-98/01 (1998).

[39] NUSEX Collaboration, M. Aglietta et al., Europhys. Lett. 8 (1989) 611;

Fréjus Collaboration, K. Daum et al., Z. Phys. C 66 (1995) 417;

Soudan II Collaboration, W.W.M. Allison et al., Phys. Lett. B 391 (1997) 491.

[40] P. Lipari, M. Lusignoli and F. Sartogo, Phys. Rev. Lett. 74 (1995) 4384.

[41] MACRO Collaboration, M. Ambrosio et al., INFN/AE-97/55, submitted to Phys. Rev. D.

[42] M. Srednicki, in Review of Particle Physics, Phys. Rev. D 54 (1996), p. 116.

[43] K.R. Lang et al., in Review of Particle Physics, Phys. Rev. D 54 (1996), p. 66.

[44] H. Harari, Phys. Lett. B 216 (1989) 413;

J. Ellis, J.L. Lopez and D.V. Nanopoulos, Phys. Lett. B 292 (1992) 189.

[45] CHORUS Collaboration, E. Eskut et al., Phys. Lett. B 424 (1998) 202;

NOMAD Collaboration, J. Altegoer et al., CERN-EP/98-57, to appear in Phys. Lett. B;

CHORUS Collaboration, E. Eskut et al., CERN-EP/98-149, submitted to Phys. Lett. B.

[46] E531 Collaboration, N. Ushida et al., Phys. Rev. Lett. 572897 (1986).

[47] CHARM-II Collaboration, M. Gruwe et al., Phys. Lett. B 309 (1993) 463;

CCFR Collaboration, K.S. McFarland et al., Phys. Rev. Lett. 75 (1995) 3993;

CDHS Collaboration, F. Dydak et al., Phys. Lett. B 134 (1984) 281.

[48] P. Vogel, in Review of Particle Physics, Phys. Rev. D 54 (1996), p. 289, and references therein.

[49] J. Bahcall, M.H. Pinsonnault, in Neutrino '96 [2], p. 56.

[50] Boone letter of intent, E. Church et al., LA-UR-97-2120 (nucl-ex/9706011) (1997);

Letter of intent, "Search for $\nu_{\mu}-\nu_{e}$ oscillations at the CERN PS", CERN-SPSC/97-21, SPSC/I 216, (1997).

[51] Borexino proposal, C. Arpesella at al., LNGS preprint (1991);

Borexino collaboration, G. Bellini et al, in TAUP '95 [52], p. 363.

[52] A. B. McDonald (SNO Collaboration), in TAUP '95, Proceedings of the 4th International Conference on Theoretical and Phenomenological Aspects of Underground Physics, Toledo, Spain, 1995, ed. by A. Morales, J. Morales, and J. A. Villar, [Nucl. Phys. B 48 (Proc. Suppl.)], p. 357; M. E. Moorhead, ibidem, p. 378.

[53] Palo Verde proposal, F. Boehm et al., Caltech 1994; F. Böhm et al., Calt-63-721. 
[54] ICARUS Collaboration, P. Benetti et al., Nucl. Instr. Methods A 327 (1993) 327; A 332 (1993) 332; P. Cennini et al., Nucl. Instr. Methods A 333 (1993) 567; A 345 (1994) 230; A 355 (1995) 355;

ICARUS-CERN-Milano Collaboration, CERN/SPSLC 96-58, SPSLC/P 304, December 1996.

[55] MINOS Collaboration, Fermilab proposal P875;

S.G. Wojcicki, in Neutrino '96 [2], p. 231.

[56] OPERA letter of intent, S. Shibuya et al., CERN-SPSC/97-24, SPSC/I 218, LNGSLOI-8-97 (1997);

Y. Suzuki (E362 (K2K) Collaboration), in Neutrino '96 [2], p. 237.

[57] TOSCA letter of intent, CERN-SPSC/97-5, SPSC/I 213 (1997);

R. A. Sidwell (COSMOS Collaboration) in Neutrino '96 [2], p. 152.

[58] K.A. Olive and D.N. Schramm, in Review of Particle Physics, Phys. Rev. D 54 (1996), p. 109, and references therein.

[59] R. Foot and R.R. Volkas, Phys. Rev. Lett. 75 (1995) 4350.

[60] R. Foot, M.J. Thomson and R.R. Volkas, Phys. Rev. D 53 (1996) 5349.

[61] R. Foot and R.R. Volkas, Phys. Rev. D 55 (1997) 5147.

[62] R. Foot and R.R. Volkas, Phys. Rev. D 56 (1997) 6653.

[63] N. Hata et al, Phys. Rev. D 55 (1997) 540. 